\title{
On the Public Economics of Annuities with
}

\section{Differential Mortality}

Antoine Bommier ${ }^{1}$, Marie-Louise Leroux ${ }^{2}$, Jean-Marie Lozachmeur ${ }^{3}$

October 15, 2009

${ }^{1}$ Toulouse School of Economics (CNRS-GREMAQ).

${ }^{2}$ FRS-FNRS, Université Catholique de Louvain and CORE.

${ }^{3}$ Corresponding author. Toulouse School of Economics (CNRS-GREMAQ and IDEI). Mailing: IDEI, 21 Allée de Brienne 31000 Toulouse, France. E-mail: jeanmarie.lozachmeur@univ-tlse1.fr. 


\begin{abstract}
This paper studies the problem of redistribution between individuals having different mortality rates. We use a continuous time model in which there are two types of individuals characterized by different survival probability paths. Individual preferences are represented by a generalized life cycle utility function which can exhibit temporal risk aversion. We successively compare utilitarian allocations when individuals exhibit temporal risk neutrality and temporal risk aversion. This problem is analyzed successively in the context of full information and asymmetric information on mortality rates.

JEL classification: H55, H23, I31.

Key words: Uncertain Lifetime, Redistribution, Annuities, Nonlinear Taxation.
\end{abstract}




\section{Introduction}

Studies on annuity and pensions usually rely on the seminal paper by Yaari (1965). This standard approach, though analytically convenient, relies on some strong assumptions on individuals preferences. In particular lifetime utilities are additively separable, which implies temporal risk neutrality. Such an assumption has major consequences. Firstly, it is found that the optimal annuity pattern is independent of the individual's mortality profile (Yaari, 1965, Levhari and Mirman, 1977, Barro and Friedman, 1977). Secondly optimal allocation of resources between individuals with different mortality takes a very simple form. As shown for example in Sheshinski (2006), a utilitarian social planner would like to equalize instantaneous levels of consumptions between individuals with different mortality profiles. Consequently, the Social Security scheme should redistribute lifecycle income from individuals with high mortality to those with low mortality.

Accounting for temporal risk aversion is of crucial importance when considering risks that have long term consequences. The risk of death being one of them, temporal risk aversion turns out to be a key aspect of individual preferences when studying intertemporal choice under uncertain lifetime (Bommier, 2008). In particular, when temporal risk aversion is introduced, Yaari's famous results vanish and the optimal consumption profile depends on individual's mortality. For a social planner this may be of importance for two reasons. First, the first best objective no longer corresponds to giving the same annuity profile to all individuals, independently of their mortality. Second, since people with different mortality look for different annuity profiles, the age profile of annuity becomes an interesting policy tool that can be used to achieve some redistribution.

The present paper emphasizes the role of temporal risk aversion when designing pensions for individuals having different mortality profiles. The question of pension design has been addressed by several papers using the 
standard additive approach, thus assuming temporal risk neutrality. This is the case in Sheshinsky (2006) and Cremer et al. (2007). Accounting for temporal risk aversion provides new perspectives and actually led us to deviate from the above studies on several aspects. Firstly, contrarily to what is found with the standard additive approach, it is not necessary to relate heterogeneity in mortality and heterogeneity in income or wealth to have non trivial results. Thus, we decided to focus on the simple case where all agents have the same financial endowments. The problems that result from the correlation between mortality and income or wealth, which were central in these two papers, are left for further contributions. Secondly, given the specificity of our approach, the policy tools that may be of interest are of different nature. In particular, we will consider the case of non linear annuity pricing, which has not attracted much attention so far. ${ }^{1}$

We consider a setting with heterogeneous individuals differing in their mortality profile and being on the verge of retirement. The low type individuals are characterized by a higher mortality rate at any age. Time is continuous and we consider that the economy is in a steady state. Individuals utility may exhibit temporal risk neutrality as well as temporal risk aversion. We study the design of annuity profiles implied by a utilitarian Social Planner. Our main results are as follows. First, when the government can observe individuals mortality profiles, the optimum leads to a pooling allocation if individuals preferences exhibit temporal risk neutrality. However, with temporal risk aversion, low type individuals should be offered a higher level of instantaneous consumption at any age with a lower consumption growth rate. Second, in a pooling optimum with temporal risk aversion, the consumption growth rate lies between the two first best ones. Third, when mortality rates

\footnotetext{
${ }^{1}$ While Sheshinsky (2006) only considers the case of linear tax on the return from annuities, Cremer et al. (2007) allow non-linear pricing of annuities. However, their nonlinear tax or subsidies are the results of differences in productivites rather than differences in longevity.
} 
are private information, the trade-off between present and future consumption should be distorted downwardly for the low type individuals. We show that a non-linear tax on annuities purchased by the low type individuals can implement this second best optimum. We finally illustrate the model using mortality rates observed for US females and males. Our results show that the tax rate on the return from annuity should increase with age; ranging from $2.5 \%$ at the age 60 up to $17.5 \%$ at the age 100 .

The rest of the paper is organized as follows. In Section 2, we present the model and the laissez-faire problem. In Section 3, we present the first best and the second best problems of the social planner. Section 4 concludes.

\section{The model}

We consider a small economy that is assumed to be in steady state. Every individuals are endowed with the same initial wealth $W_{0}$. The population is divided into two categories. Individuals of type $H$ are characterized by lower mortality rates than individuals of type $L$. Denoting $\mu^{H}(t)$ and $\mu^{L}(t)$ agents $H$ and $L$ hazard rate of death at age $t$, we thus assume that:

$$
A 1: \mu^{H}(t)<\mu^{L}(t) \text { for every } t
$$

Agents of type $H$ have therefore higher survival probability that agents of type $L$. We also assume that mortality rates increase with age:

$$
A 2: \frac{d}{d t} \mu^{i}(t)>0
$$

Demographic studies indicate that this assumption is realistic when considering ages greater than 25 or 30 . Since our paper deals with pensions that are 
typically received after retirement, such an assumption is rather unrestrictive. Assumption $A_{3}$ further states that the hazard rate of death increases more slowly for individuals of type $L$ :

$$
A 3: \frac{\mu^{H}(t+\varepsilon)}{\mu^{H}(t)} \geqslant \frac{\mu^{L}(t+\varepsilon)}{\mu^{L}(t)}
$$

for any $t$ and $t+\varepsilon$, where $\varepsilon>0$. In other words, this assumption states that the relative difference between mortality rates is decreasing with age. Again this assumption is supported by studies on differential mortality at adult and old ages (Brown et al., 2002).

The proportion of type $i$ individual is $n^{i}$. At each time a type $i$ individual dies, he is replaced by an individual of the same type. In all the paper, we assume that private savings are invested in perfect annuities i.e. the annuity market can distinguish the individuals types. We finally denote $j(t)$, the return on private savings at time $t$, the actuarially fair return on annuity being $j(t)+\mu^{H}(t)$ for type $H$ individuals and $j(t)+\mu^{L}(t)$ for type $L$ individuals. In the following, we present the utility function and compare some of its useful properties relative to the individuals types. Finally we present the Laissez Faire problem.

\subsection{Individual preferences}

Yaari's standard approach consists in assuming a life of length $T$ with a consumption profile $c($.$) that yields a lifetime utility:$

$$
U^{\text {yaari }}(c, T)=\int_{0}^{T} \alpha(t) u(c(t)) d t
$$

where $\alpha(t)$ is an exogenous time discount factor. Bommier (2006) emphasized the limits of such an approach which relies on the assumption of tem- 
poral risk neutrality, a rather unappealing assumption for dealing with risk which have long term consequences, such as the risk of death. Temporal risk aversion can be introduced, without abandoning the expected utility framework, by considering utility functions of the form:

$$
U(c, T)=\phi\left(\int_{0}^{T} \alpha(t) u(c(t)) d t\right)
$$

where $\phi$ is an increasing function. As is known from Kihlstrom and Mirman (1974), playing the function $\phi$ involves adjusting individuals risk aversion. When $\phi$ is concave the agent with the above utility function is simply more risk averse than the agent with Yaari's utility function (which is obtained when $\phi$ is linear).

A simple interpretation of the specification in (1) is that agents have a linear "lifetime felicity". Each moment of life gives them an instantaneous felicity $\alpha(t) u(c(t))$ that is additively aggregated in order to get the lifetime felicity. However, given the uncertainty about life duration (and about consumption), individuals cannot know ex-ante what will their lifetime felicity be. At most, they know the distribution of lifetime felicity. Introducing a function $\phi$ as in (2) enables to consider risk aversion with respect to lifetime felicity. For consumption profiles that would provide a constant flow of felicity, the function $\phi$ would determine individual risk aversion with respect to life duration. A linear $\phi$ would involve assuming risk neutrality, while a concave function $\phi$ would indicate a positive risk aversion. While there is no theoretical obstacle to considering risk prone agents, we limit ourselves to the case where $\phi$ is concave $\left(\phi^{\prime \prime} \leq 0\right)$ and where $-\phi^{\prime \prime} / \phi^{\prime}$ is a non increasing function (consistent with the idea of non increasing absolute risk aversion with respect to lifetime felicity).

There is no possible dispute about the fact that introducing temporal risk aversion complicates the computation associated with utility maximization. 
Actually, this is probably one of the main reasons that led economists to focus on Yaari's specification for so many years. A major difficulty seems to appear when writing the expected utility function. Indeed, when life duration is random, the expected lifetime utility associated with a given a consumption profile is:

$$
E U(c)=\int_{0}^{+\infty} \mu(t) \exp \left(-\int_{0}^{t} \mu(\tau) d \tau\right) \phi\left(\int_{0}^{t} \alpha(\tau) u(c(\tau)) d \tau\right) d t
$$

By integration by part, this may also rewrite:

$$
E U(c)=\int_{0}^{+\infty} s(t) \alpha(t) u(c(t)) \phi^{\prime}\left(\int_{0}^{t} \alpha(\tau) u(c(\tau)) d \tau\right) d t
$$

where $s(t)=\exp \left(-\int_{0}^{t} \mu(\tau) d \tau\right)$ is the probability of being alive at age $t$. When $\phi$ is not linear, expected utility is then no longer additive, which might look like the beginning of a nightmare for economists. Bommier (2006) explains however that this difficulty can easily be avoided by making a linear approximation. This allows to avoid the pangs of endogenous discounting without loosing most of the insights brought by this novel approach.

The idea is to rely on what is called the assumption of a "priceless life context". Basically, this assumption consists in assuming that the difference in terms of welfare between life and death is much greater than the difference between high and low levels of consumption. Under this assumption (and through an appropriate renormalization of the functions $\phi$ and/or $\alpha$ ), preferences can be approximated by an additive expected lifetime utility function: ${ }^{2}$

$$
E U(c)=\int_{0}^{T} s(t) \alpha(t) \beta(t) u(c(t)) d t
$$

\footnotetext{
${ }^{2}$ See appendix A for computations.
} 
where

$$
\beta(t)=\frac{-1}{s(t)} \int_{t}^{T} \dot{s}(\tau) \phi^{\prime}\left(\int_{0}^{\tau} u_{0} \alpha(x) d x\right) d \tau
$$

The main departure from Yaari comes from $\beta(t)$ which constitutes the time discounting factor. Note that when $\phi$ is linear, as in Yaari's case, $\beta(t)$ is constant and can be omitted. In the other cases, however, $\beta$ is not constant and its shape depends both on the mortality risks (through the survival function $s(t))$ and on the degree of temporal risk, via the function $\phi$. When $\phi$ is concave, $\beta$ is decreasing, reflecting the fact that the combination of temporal risk aversion with mortality risks generate time discounting as explained in details in Bommier (2006). We finally assume in the rest of the paper that the utility function $u($.$) exhibits a constant intertemporal elasticity of$ substitution that is $c u^{\prime \prime}(c) / u^{\prime}(c)$ is a constant.

\subsection{Individuals types and preferences properties}

Before going further, it is useful to compare both types of individuals preferences properties. We prove the following lemma in the appendix.

Lemma 1 If individuals mortality patterns satisfy assumptions A1-A3, then at any times $t$ and $t+\varepsilon, \beta^{H}(t) \leq \beta^{L}(t)$ and $\beta^{H}(t+\varepsilon) / \beta^{H}(t) \geq \beta^{L}(t+\varepsilon) / \beta^{L}(t)$.

This lemma tells that time discounting generated by mortality profiles are such that individuals of type $L$ value more consumption at any date. Furthermore, the time discount factor decreases at a higher rate for low survival individuals.

Note finally that assumptions A1 to A3 imply some monotonicity properties on individuals indifference curves. To see this write the marginal rate of substitution between consumptions at date $t$ and $t+\varepsilon$ for any pair of 
consumptions $(c(t), c(t+\varepsilon))$. Differentiation of $(3)$ gives:

$$
\begin{aligned}
M R S_{c(t), c(t+\varepsilon)}^{i} & =\left.\frac{d c(t)}{d c(t+\varepsilon)}\right|_{E U^{i}} \\
& =-\frac{s^{i}(t+\varepsilon) \alpha(t+\varepsilon) \beta^{i}(t+\varepsilon) u^{\prime}(c(t+\varepsilon))}{s^{i}(t) \alpha(t) \beta^{i}(t) u^{\prime}(c(t))}
\end{aligned}
$$

A2 implies $s^{L}(t+\varepsilon) / s^{L}(t)<s^{H}(t+\varepsilon) / s^{H}(t)$ and A3 implies $\beta^{L}(t+\varepsilon) / \beta^{L}(t)<$ $\beta^{H}(t+\varepsilon) / \beta^{H}(t)$ so that $M R S_{c(t), c(t+\varepsilon)}^{H}<M R S_{c(t), c(t+\varepsilon)}^{L}$. In other words, the slope of indifference curve in the $\{c(t+\varepsilon), c(t)\}$ space is less steeper for the type $L$ individuals. It just says that for a given decrease in future consumption, low type individuals should be more compensated in term of present consumption than high type individuals. This is illustrated in figure 1:

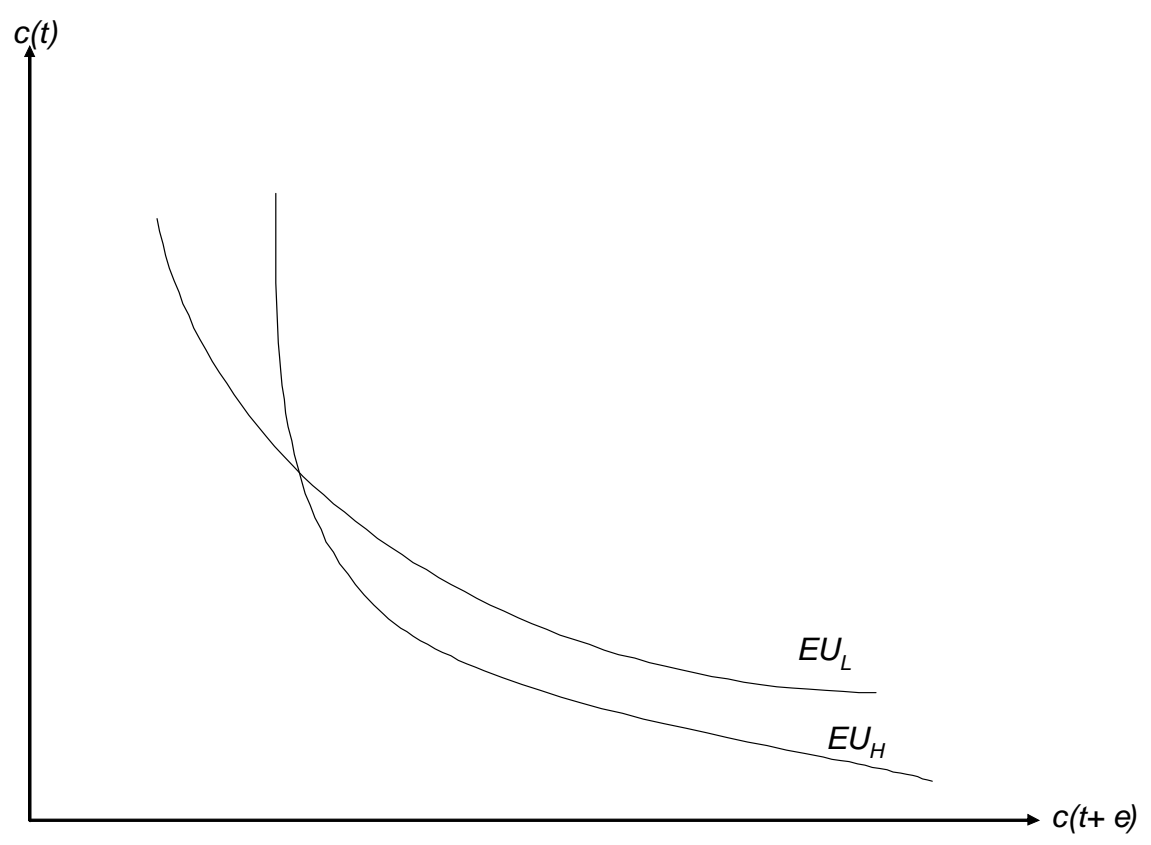

Figure 1: Indifference curves in the $\{c(t+\varepsilon), c(t)\}$ space. 


\subsection{The individual's problem and the laissez faire}

We assume a perfect annuity market. Individuals can buy annuities and the after-tax rate of interest of these annuities at time $t$ is $r(t)$. Reintroducing upperscripts to distinguish individuals types, individual $i$ 's problem is thus:

$$
\begin{aligned}
& \max _{c(t)} E U^{i}(c)=\int_{0}^{T} s^{i}(t) \alpha(t) \beta^{i}(t) u(c(t)) d t \\
& \text { s.to } \int_{0}^{T} \exp \left(\int_{0}^{t}-r(\tau) d \tau\right) c(t) d t \leq W_{0} .
\end{aligned}
$$

The first order condition of the individual's problem is given by:

$$
s^{i}(t) \beta^{i}(t) \alpha(t) u^{\prime}\left(c^{i}(t)\right)=\lambda \exp \left(-\int_{0}^{t} r(\tau) d \tau\right)
$$

where $\lambda$ is the Lagrange multiplier associated with the individual's budget constraint. Note that when annuity prices are fair, $r(t)=j(t)+\mu(t),(6)$ can be rewritten as:

$$
\beta^{i}(t) \alpha(t) u^{\prime}\left(c^{i}(t)\right)=\lambda \exp \left(-\int_{0}^{t} j(\tau) d \tau\right)
$$

where $j(t)$ is the interest rate. It can then be shown that: ${ }^{3}$

Proposition 1 When the return of the annuity market is actuarially fair and under assumptions A1-A3, the laissez faire allocation is such that:

(a) With temporal risk neutrality (i.e. when $\phi$ is linear): $\dot{c}^{L}(t) / c^{L}(t)=$ $\dot{c}^{H}(t) / c^{H}(t)$ for every $t$.

(b) With temporal risk aversion (i.e. when $\phi$ is concave): $\dot{c}^{L}(t) / c^{L}(t)<$ $\dot{c}^{H}(t) / c^{H}(t)$ for every $t$.

\footnotetext{
${ }^{3}$ See appendix $C$.
} 
When temporal risk aversion is introduced, type $H$ and $L$ agents choose different consumption path. Actually, agent $H$ whose mortality is low chooses a higher rate of consumption growth (or a lower rate of consumption decline) than agent $L$. This reflects the relation between mortality and impatience discussed in Bommier (2008). Since agents' optimal strategies are different, we may anticipate that a social planner may be willing to provide different pension levels and different pension profiles to individuals of different types. Moreover, in the case where the type is not observable, the planner may use this heterogeneity of individuals' optimal strategies to make them reveal their type by letting them choose a pension plan among several alternatives. We address these questions below where we discuss the planner's optimal strategy, depending on whether individuals' mortality is private information or not.

Note finally for further reference in section 3.2 that (6) can be rewritten in terms of marginal rate of substitution as follows:

$$
M R S_{c(t), c(t+\varepsilon)}^{i}=-\exp \left(-\int_{t}^{t+\epsilon} r(\tau) d \tau\right)
$$

where $M R S_{c(t), c(t+\varepsilon)}^{i}$ is given by (5).

\section{The optimal policy}

\subsection{Full information}

Assume first that the government is utilitarian and can perfectly observe the individuals' types. In this first best problem, the social planner is maximizing the sum of individuals' expected utility functions under the resource 
constraint of the economy. Its problem is thus:

$$
\begin{array}{cc} 
& \max _{c^{H}(t), c^{L}(t)} n^{H} E U^{H}\left(c^{H}\right)+n^{L} E U^{L}\left(c^{L}\right) \\
\text { s.to } & \int_{0}^{T} n^{H} s^{H}(t) \exp \left(\int_{0}^{t}-j(\tau) d \tau\right) c^{H}(t) d t \\
+ & \int_{0}^{T} n^{L} s^{L}(t) \exp \left(\int_{0}^{t}-j(\tau) d \tau\right) c^{L}(t) d t \leq W_{0}
\end{array}
$$

The first order conditions of the first best problem are:

$$
\beta^{i}(t) \alpha(t) u^{\prime}\left(c^{i}(t)\right)=\lambda \exp \left(-\int_{0}^{t} j(\tau) d \tau\right)=0
$$

for any $i=H, L$ and every $t$ and $\lambda$ is the Lagrange multiplier associated with the resource constraint. We prove the following proposition in the appendix: Proposition 2 With assumptions A1-A2, the first best allocation is characterized by:

(a) With temporal risk neutrality, $c^{H}(t)=c^{L}(t) \forall t$.

(b) With temporal risk aversion on the length of life:

(i) $c^{H}(t)<c^{L}(t)$ for every $t$.

(ii) With assumption A3, $\dot{c}^{L}(t) / c^{L}(t)<\dot{c}^{H}(t) / c^{H}(t)$ for every $t$.

Under the assumption of temporal risk neutrality, point (a) of proposition 2 states that the optimum is pooling every individuals with the same levels of consumption across individuals and time. However, as stressed in point (b), when individuals expected utility exhibit temporal risk aversion, the optimum is to offer a higher instantaneous consumption level for the low survival individuals at every time. This is because, low type individuals have on average a lower lifetime felicity and that lifetime utility is concave in lifetime felicity. As in the laissez faire however, the consumption level of type $H$ individuals decreases at a lower rate. In case (a), it is clear that there is a transfer 
of (expected) lifetime income from the low to the high type individuals. The

level of this transfer is measured by $\left(\int_{0}^{\infty} n^{H} s^{H}(t) c(t) d t-\int_{0}^{\infty} n^{L} s^{L}(t) c(t) d t\right)$ where $c(t)$ is the optimal consumption profile for both types of individuals. In case $(b)$ however, whether there is a positive or negative transfer of life cycle income from one type to the other is ambiguous.

To decentralize this first best optimum, one only needs lump sum transfers from one type to the other and fair annuity prices. To see this, observe that equation (9) is equivalent to its laissez faire counterpart (7) where annuity prices are actuarially fair.

\subsection{Asymmetric information}

Assume now that the government is unable to tell who is of type $L$ and who is of type $H$. Such a government may have different strategies. A first possibility is to look for a pooling optimum. Another consists in offering a menu of pension, so that individuals would voluntarily reveal their type.

\subsubsection{Pooling optimum}

Let's assume that the government now is constrained to offer the same instantaneous consumption $c(t)$ to both types of individuals. His problem writes:

$$
\begin{aligned}
& \max _{c(t)} n^{H} E U^{H}(c)+n^{L} E U^{L}(c) \\
& \text { s.to } \int_{0}^{\infty}\left(n^{H} s^{H}(t)+n^{L} s^{L}(t)\right) \exp \left(\int_{0}^{t}-j(\tau) d \tau\right) c(t) d t \leq W_{0}
\end{aligned}
$$


The first order condition with respect to $c(t)$ yields:

$$
\bar{\beta}(t) \alpha(t) u^{\prime}(c(t))=\lambda \exp \left(\int_{0}^{t}-j(\tau) d \tau\right)
$$

where $\lambda$ is the Lagrange multiplier associated with the resource constraint and $\bar{\beta}(t)=\left[n^{H} s^{H}(t) \beta^{H}(t)+n^{L} s^{L}(t) \beta^{L}(t)\right] /\left[n^{H} s^{H}(t)+n^{L} s^{L}(t)\right] \cdot \bar{\beta}(t)$ is a weighted sum of the $\beta^{i}(t)$ 's with the weight given on $\beta^{i}(t)$ by the fraction of individuals of type $i$ surviving at period $t: n^{i} s^{i}(t) / \sum_{j=H, L} n^{j} s^{j}(t)$. In the appendix, we prove that $\dot{\beta}^{L}(t) / \beta^{L}(t)<\bar{\beta}(t) / \bar{\beta}(t)<\dot{\beta}^{H}(t) / \beta^{H}(t)$ for every t. This implies that:

$$
\left(\frac{\dot{c}^{L}(t)}{c^{L}(t)}\right)^{F B}<\frac{\dot{c}(t)}{c(t)}<\left(\frac{\dot{c}^{H}(t)}{c^{H}(t)}\right)^{F B}
$$

for every $t$ and where $F B$ stands for the first best allocation. In other words, the variation rate of the consumption profile in the pooling optimum lies between the First Best variation rates in the First Best optimum.

\subsubsection{Second best}

Now we look for the case where the planner can offer different annuity profiles. Beacause, of point $(i)$ of proposition 2, there is no adverse selection since the optimum is to pool individuals with theh same level of instaneous consumption. However, with temporal risk aversion, it is clear that individuals of type $H$ would like to mimic individuals of type $H$. The problem of the government is the first best problem to which we add an incentive compatible constraint stating that type $H$ individuals do not get a lower utility if they 
reveal their true type:

$$
\begin{array}{ll} 
& \max _{c^{H}(t), c^{L}(t)} n^{H} E U^{H}\left(c^{H}\right)+n^{L} E U^{L}\left(c^{L}\right) \\
\text { s.to }: & \int_{0}^{\infty} n^{H} s^{H}(t) \exp \left(\int_{0}^{t}-j(\tau) d \tau\right) c^{H}(t) d t+ \\
& \int_{0}^{\infty} n^{L} s^{L}(t) \exp \left(\int_{0}^{t}-j(\tau) d \tau\right) c^{L}(t) d t \leq W_{0}, \\
& E U^{H}\left(c^{H}\right) \geq E U^{H}\left(c^{L}\right) .
\end{array}
$$

Denoting $\gamma$ the Lagrange multiplier associated to the incentive compatibility constraint and $\pi(t)=\left[\gamma / n^{L}\right]\left[s^{H}(t) \beta^{H}(t)\right] /\left[s^{L}(t) \beta^{L}(t)\right]>0$, the first order conditions yield:

$$
\begin{aligned}
M R S_{c(t), c(t+\varepsilon)}^{H}= & -\exp \left(-\int_{t}^{t+\varepsilon}\left(j(\tau)+\mu^{H}(\tau)\right) d \tau\right) \\
M R S_{c(t), c(t+\varepsilon)}^{L}= & -\exp \left(-\int_{t}^{t+\varepsilon}\left(j(\tau)+\mu^{L}(\tau)\right) d \tau\right) \\
& \times\left[\frac{1-\pi(t)}{1-\pi(t) \frac{\overline{M R S}_{c(t), c(t+\varepsilon)}^{H}}{M R S_{c(t), c(t+\varepsilon)}^{L}}}\right]
\end{aligned}
$$

for any $t$ and $t+\varepsilon$ and where $\overline{M R S}_{c(t), c(t+\varepsilon)}^{H}$ is the marginal rate of substitution of a type $H$ individual mimicking a type $L$ individual.

Comparing (11) with (8), we obtain the usual result of no distortion at the top, which means that the first best trade-off between two-period consumptions is preserved for the high survival individuals. On the contrary, the second best optimum introduces a distortion in the trade-off between two-period consumptions for the low type individual. As shown in section 2.2, $M R S_{c(t), c(t+\varepsilon)}^{H}<M R S_{c(t), c(t+\varepsilon)}^{L}$ for the same pair of consumption $\{c(t), c(t+\varepsilon)\}$. Thus $\overline{M R S}_{c(t), c(t+\varepsilon)}^{H} / M R S_{c(t), c(t+\varepsilon)}^{L}>1$ and the expression 
in brackets of (12) is greater than one. We summarize these results in the following proposition: ${ }^{4}$

Proposition 3 With assumptions A1-A3, the second best allocation is characterized by:

(i) With risk neutrality on the length of life, the first best solution is implementable.

(ii) With risk aversion on the length of life, the second best solution is characterized by:

(a) $M R S_{c(t), c(t+\epsilon)}^{H}=-\left[s^{H}(t+\varepsilon) / s^{H}(t)\right] \exp \left(-\int_{t}^{t+\varepsilon} j(\tau) d \tau\right)$ for any $t$ and $\varepsilon>0$.

(b) $M R S_{c(t), c(t+\epsilon)}^{L}<-\left[s^{L}(t+\varepsilon) / s^{L}(t)\right] \exp \left(-\int_{t}^{t+\varepsilon} j(\tau) d \tau\right)$ for any $t$ and $\varepsilon>0$.

(c) $\left(\dot{c}^{L}(t) / c^{L}(t)\right)^{S B}<\left(\dot{c}^{L}(t) / c^{L}(t)\right)^{F B}<\left(\dot{c}^{H}(t) / c^{H}(t)\right)^{S B}=\left(\dot{c}^{H}(t) / c^{H}(t)\right)^{F B}$

where $F B$ and $S B$ stand respectively for the first and the second best allocations.

As argued above, the first best solution is incentive compatible when individuals are risk neutral towards the length of life. When risk averse, point $i$ (a) states that the consumption path of individuals of type $H$ is not distorted. This is the usual "no distortion at the top" result. However, for type $L$ individuals, the marginal rate of substitution between present and future consumption is distorted downwardly. In words, it is desirable to encourage early consumption in life as compared to the first best trade-off. Intuitively, this property can be explained by the fact that type $L$ individuals have steeper indifference curves in the $\{c(t+\varepsilon), c(t)\}$ space. This implies that, starting from the first best trade-off, a variation $d c(t+\varepsilon)<0$ along with a variation $d c(t)=M R S_{c(t), c(t+\varepsilon)}^{L} d c(t+\varepsilon)>0$ has no first order effect on the utility of type $L$ individuals while it decreases the life cycle utility

\footnotetext{
${ }^{4}$ See the appendix for details.
} 
of type $H$ individuals. This distortion is thus a way to relax an otherwise binding self selection constraint. As a result, point (c) stresses that the variation rate of consumption of type $L$ individuals is lower than the one in the first best. As discussed in the following, this optimum is compatible with a tax on the return from annuities for the type $L$ individuals. We present an implementation procedure with such a tax in the following section.

\subsubsection{Implementation of the second best}

This section shows how one can implement the second best allocation described in proposition 3. Since there is no distortion for type $H$ individuals, no tax on its actuarially fair return on annuities is desirable. For type $L$ individuals the instantaneous return on annuities is $r^{L}(t)$. Thus if the tax on the return of annuities is $\rho^{L}(t)$, then $r^{L}(t)=\left(1-\rho^{L}(t)\right)\left(j(t)+\mu^{L}(t)\right)$. In order to decentralize the second best optimum, the Laissez Faire solution chosen by the individual of type $L$ should coincide with the second best allocation. Thus $r^{L}(t)$ is obtained by equating the RHS of (8) and (12) which yields:

$\exp \left(-\int_{t}^{t+\epsilon} r^{L}(\tau) d \tau\right)=\left[\frac{1-\pi(t)}{1-\pi(t) \frac{\overline{M R S}_{c(t), c(t+\epsilon)}}{M R S_{c(t), c(t+\epsilon)}^{L}}}\right] \exp \left(\int_{t}^{t+\varepsilon}-\left(j(\tau)+\mu^{L}(\tau)\right) d \tau\right)$

Note that for $\epsilon$ small, one has:

$$
M R S_{c(t), c(t+\epsilon)}^{i}=1+\epsilon\left[\frac{\dot{s}^{i}(t)}{s^{i}(t)}+\frac{\dot{\alpha}(t)}{\alpha(t)}+\frac{\dot{\beta}^{i}(t)}{\beta^{i}(t)}+\frac{u^{\prime \prime}(c(t))}{u^{\prime}(c(t))} \dot{c}(t)\right]
$$


We show in the appendix that (13) together with (14) yields for $\epsilon$ small:

$$
r^{L}(t)-\left(j(t)+\mu^{L}(t)\right)=\frac{\pi(t)}{1-\pi(t)}\left[\frac{\dot{s}^{L}(t)}{s^{L}(t)}-\frac{\dot{s}^{H}(t)}{s^{H}(t)}+\frac{\dot{\beta}^{L}(t)}{\beta^{L}(t)}-\frac{\dot{\beta}^{H}(t)}{\beta^{H}(t)}\right]
$$

so that the tax rate on the actuarially fair return on annuities is given by:

$$
\rho^{L}(t)=\left(\frac{1}{\left(j(t)+\mu^{L}(t)\right)}\right)\left(\frac{\pi(t)}{1-\pi(t)}\right)\left[\frac{\dot{s}^{H}(t)}{s^{H}(t)}-\frac{\dot{s}^{L}(t)}{s^{L}(t)}+\frac{\dot{\beta}^{H}(t)}{\beta^{H}(t)}-\frac{\dot{\beta}^{L}(t)}{\beta^{L}(t)}\right]
$$

which is always positive by assumption A2 and lemma 1. The second best allocation is thus implementable with an age dependent tax on the return of annuities given by (15) and some transfers between the two types of individuals. However, given the complexity of the formula, it is highly complicated to derive the properties of the tax rate with respect to $t$. The following part presents a numerical simulation of the model in order to have an idea about the shape and the level of this tax function.

\subsubsection{Numerical simulation}

For this numerical part, we take a population aged more than 60 . This corresponds to the case in which individuals are endowed with a certain amount of capital $W$ and decide to annuitize it at the age of 60 . Our types of individuals $H$ and $L$ have mortality rates similar to US women and men born in the 40's. We did not choose these gender specific mortality to provide conclusion on gender issues. Actually, gender is generally well observed by the social planner and, therefore, not associated with the problem of asymmetric information that motivated the second best approach. We only used these male and female mortality because they provide mortality rates and a differential 
mortality that are of a reasonable order of magnitude. It is assumed that $n_{H}=n_{L}=0.5$. We calibrate the mortality patterns with a Gompertz law which is a generally accepted distribution of mortality after the age of 40 . Formally, the Gompertz law is written as:

$$
\mu^{i}(t)=\mu_{0}^{i} \exp (k t)
$$

We took $k=0.08$, which corresponds to a rather consensual value in demographic studies. The parameters $\mu_{0}^{i}$ are found by equating observed mortality rates at 60 to $0.76 \%$ for women and $1.12 \%$ for men. ${ }^{5}$ We further assume that the subjective discount factor is such that $\alpha(x)=1$ and the gross rate of interest is $3 \%$. In order to calibrate the objective discount factor, we use a function $\phi(x)=[1-\exp (-a x)]$ and parametrize $a$ such that $-\dot{\beta}^{L}(60) / \beta^{L}(60)=0.03$. In other words, we assume that the total rate of discount is equal to the gross interest rate at the age 60 for type $L$ individuals. Finally, the utility function is given by the following CRRA utility function:

$$
U(c)=1+\frac{c^{1-\sigma}}{1-\sigma}
$$

where $\sigma=0.8$.

In the First Best optimum, the results suggest that type $L$ individuals are net recipient of the transfer scheme. Their expected income is equal to $54.9 \%$ of the total income. In the second best optimum however, their expected income falls to $47.3 \%$ of the total income thus making them net contributors to the transfer scheme. The welfare increases by $5.7 \%$ from the Laissez Faire to the Second Best. The figure 2 represents the tax rate on the return of annuities obtained for type $L$ individuals as a result of the second best allocation presented in the preceding section. It can be seen that this

\footnotetext{
${ }^{5}$ These data come from the Berkeley Mortality Database.
} 
tax rate is monotonically increasing with age. It goes from $2.5 \%$ at the age 60 to more than $17.5 \%$ at the age 100 .

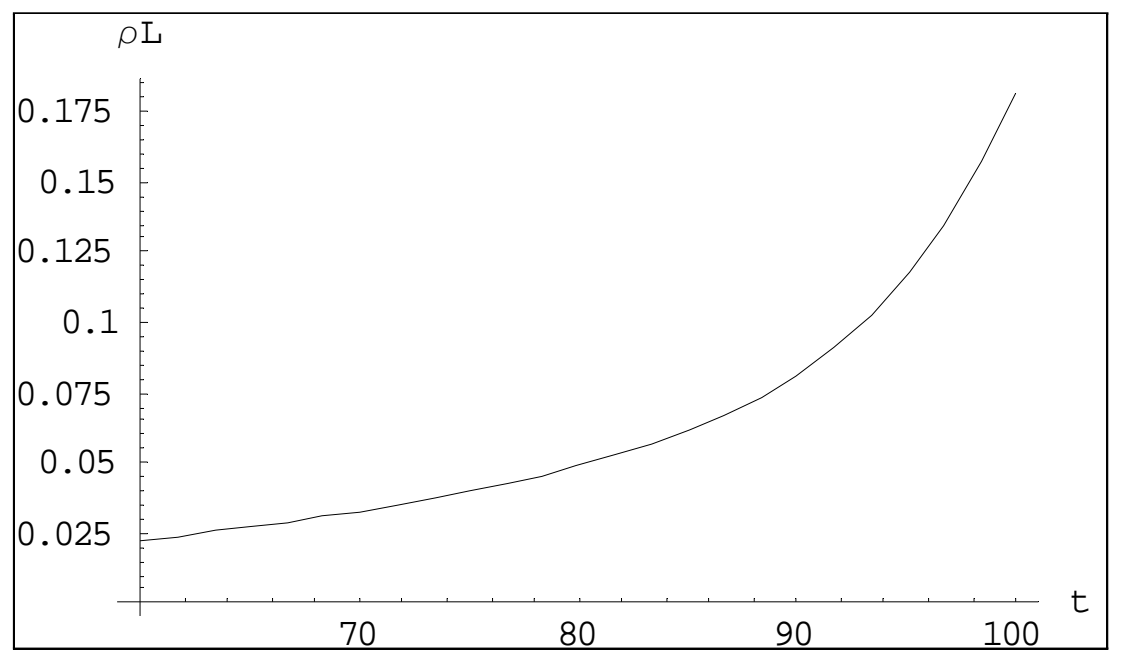

Figure 2: The annuity tax rate on type $L$ individuals

\section{Conclusion}

This paper has studied the problem of redistribution between individuals differing in their survival probabilities. We have compared successively utilitarian allocations when individuals are either temporal risk neutral or temporal risk averse. In a first best setting, we find that if individuals are temporal risk averse, long-lived individuals should have a lower instantaneous consumption than the one of short-lived. On the contrary, with temporal risk neutrality, the pooling allocation is socially optimal. When the government cannot observe individuals risk of death, the first best allocation is implementable with no distortion on the price of fair annuities and a transfer from the low survival to the high survival individuals. With temporal risk aversion, we find no distortion in the trade-off between two-period instantaneous consumptions for the high-type individual. However consumption in earlier periods of life is encouraged for the low-type individual. Consumption for this individual is also decreasing faster in a second best than in a first best framework. We 
finally study how to decentralize these allocations through the annuity market. We show that it is optimal to distort the price of annuities from the actuarially fair price. For the low-survival individuals, a tax on the return from annuities turns out to be desirable. A numerical illustration based on gender mortality differences shows that the tax rate on annuity return should increase with age ranging from $2.5 \%$ at age 60 to $17.5 \%$ at age 100 .

\section{References}

[1] Barro, R. J, and J. W. Friedman, 1977, "On Uncertain Lifetimes". The Journal of Political Economy, 85(4):843-849.

[2] Berkeley Mortality Database, http://www.demog.berkeley.edu/ bmd/states.html.

[3] Bommier, A., 2006. Uncertain lifetime and intertemporal choice: risk aversion as a rationale for time discounting. International Economic Review 47(4), 1223-1246.

[4] Bommier, A., 2008, Rational Impatience. Working Paper university of Toulouse.

[5] Bommier, A., Magnac, T., Rapoport, B., Roger, M., 2006. Droit à la retraite et mortalité différentielle. Economie et Prévision 168, 1-16.

[6] Brown, J. R., Liebman J. B., and J. Pollet, 2002, "Estimating Life Tables That Reflect Socioeconomic Differences in Mortality", in The Distributional Aspects of Social Security and Social Security Reform, M. Feldstein and J. B. Liebman, eds, The University of Chicago Press.

[7] Coronado, J. L., D. Fullerton and T. Glass, 2000. The progressivity of Social Security. NBER Working papers 7520. 
[8] Cremer, H., J.M Lozachmeur and P. Pestieau, 2007. Collective annuities and redistribution. CORE Discussion Paper 2007/95.

[9] Kihlstrom, R.E. and L.J. Mirman, 1974, "Risk Aversion with many Commodities". Journal of Economic Theory 8:361-88.

[10] Levhari, D. and L. J. Mirman, 1977, "Savings and Consumption with an Uncertain Horizon". The Journal of Political Economy, 85(2):265-281.

[11] Liebman, J.B., 2001. Redistribution in the current U.S Social Security system. NBER Working Papers 8625.

[12] Sheshinski, E., 2007. Utilitarian pricing of annuities, Chapter 13 in "The economic theory of annuities". Princeton University Press.

[13] Simonovits A., 2006. Optimal Design of Pension Rule with Flexible Retirement: The Two-Type Case. Journal of Economics 89 (3), 197-222.

[14] Yaari, M., 1965. Uncertain lifetime, life insurance and the theory of the consumer. The Review of Economic Studies, 32, 2,137-150.

\section{Appendix}

\section{A Construction of the expected lifetime util-}

\section{ity}

To be able to get back to a simple additive specification, we make the assumption of a "priceless life context". As defined in Bommier (2006), it corresponds to a situation where $u(c(t))=1+\lambda \omega(c(t))$ where $\lambda$ is a (small) scalar and $\omega($.$) is bounded. The lifetime expected utility function can then$ 
be rewritten as:

$$
\begin{aligned}
& E U(c)=\int_{0}^{T} s(t) \alpha(t) \phi^{\prime}\left(\int_{0}^{t} \alpha(x)(1+\lambda \omega(c(x))) d x\right) d t \\
& +\lambda \int_{0}^{T} s(t) \alpha(t) \omega(c(t)) \phi^{\prime}\left(\int_{0}^{t} \alpha(x)(1+\lambda \omega(c(x))) d x\right) d t .
\end{aligned}
$$

We assume that $\lambda \rightarrow 0$ which means that the individual would agree to give up most of his consumption to live longer. Taking the Taylor expansion of the function $\phi^{\prime}$ and keeping only the terms of order zero and one in $\lambda$, this yields:

$$
\begin{aligned}
& E U(c)=\int_{0}^{T} s(t) \alpha(t) \phi^{\prime}\left(\int_{0}^{t} \alpha(x) d x\right) d t \\
& +\lambda \int_{0}^{T} s(t) \alpha(t) \omega(c(t)) \phi^{\prime}\left(\int_{0}^{t} \alpha(x) d x\right) d t \\
& +\lambda \int_{0}^{T} s(t) \alpha(t)\left(\int_{0}^{t} \alpha(x) \omega(c(x)) d x\right) \phi^{\prime \prime}\left(\int_{0}^{t} \alpha(x) d x\right) d t .
\end{aligned}
$$

Denoting the constant $\Psi=\int_{0}^{T} s(t) \alpha(t) \phi^{\prime}\left(\int_{0}^{t} \alpha(x) d x\right) d t$ and switching the order of integration of the third term, the expected utility function can be approximated by:

$$
\begin{aligned}
E U(c) \approx & \Psi+\lambda \int_{0}^{T} s(t) \alpha(t) \omega(c(t)) \phi^{\prime}\left(\int_{0}^{t} \alpha(x) d x\right) d t \\
& +\lambda \int_{0}^{T} \alpha(t) \omega(c(t))\left(\int_{t}^{+\infty} s(x) \alpha(x) \phi^{\prime \prime}\left(\int_{0}^{x} \alpha(\tau) d \tau\right) d x\right) d t \\
\approx & \Psi+\lambda \int_{0}^{T} \alpha(t) \omega(c(t))\left[\begin{array}{c}
s(t) \phi^{\prime}\left(\int_{0}^{t} \alpha(x) d x\right)+ \\
\int_{t}^{T} s(x) \alpha(x) \phi^{\prime \prime}\left(\int_{0}^{x} \alpha(\tau) d \tau\right) d x
\end{array}\right] d t .
\end{aligned}
$$


Integrating by part the term in brackets yields:

$$
E U(c) \approx \Psi+\lambda \int_{0}^{T} s(t) \alpha(t) \omega(c(t)) \beta(t) d t
$$

where $\beta(t)=\int_{t}^{T}-(\dot{s}(\tau) / s(t)) \phi^{\prime}\left(\int_{0}^{\tau} \alpha(x) d x\right) d \tau$. Using $\omega(c(t))=u(c(t))-$ $1 / \lambda$ and forgetting the constant, the expected lifetime utility can thus be approximated by the following additive utility function:

$$
E U(c)=\int_{0}^{T} s(t) \alpha(t) \beta(t) u(c(t)) d t
$$

Finally, denoting respectively $\dot{\beta}(t)$ and $\dot{s}(t)$ the derivatives of $\beta(t)$ and $s(t)$ with respect to $t$ yields:

$$
\begin{aligned}
\dot{\beta}(t) & =\frac{\dot{s}(t)}{s(t)^{2}} \int_{t}^{T} \dot{s}(\tau) \phi^{\prime}\left(\int_{0}^{\tau} \alpha(x) d x\right) d \tau+\frac{\dot{s}(t)}{s(t)} \phi^{\prime}\left(\int_{0}^{t} \alpha(x) d x\right) \\
& =-\frac{\dot{s}(t)}{s(t)} \int_{t}^{T} \frac{s(\tau)}{s(t)} \phi^{\prime \prime}\left(\int_{0}^{\tau} \alpha(x) d x\right) d \tau
\end{aligned}
$$

where we made use of integration by-part.

\section{B Proof of Lemma 1}

Let's denote $\Delta(t)=-\dot{\beta}^{L}(t) / \beta^{L}(t)-\left(-\dot{\beta}^{H}(t) / \beta^{H}(t)\right)$. We want to show that this term is positive for any $t$. Using the definition of $\mu(t)$, one has:

$\Delta(t)=\frac{\mu^{L}(t) \int_{t}^{T} \frac{s^{L}(\tau)}{s^{L}(t)}\left(-\phi^{\prime \prime}\left(\int_{0}^{\tau} \alpha(x) d x\right)\right) d \tau}{\int_{t}^{T} \mu^{L}(\tau) \frac{s^{L}(\tau)}{s^{L}(t)} \phi^{\prime}\left(\int_{0}^{\tau} \alpha(x) d x\right) d \tau}-\frac{\mu^{H}(t) \int_{t}^{T} \frac{s^{H}(\tau)}{s^{H}(t)}\left(-\phi^{\prime \prime}\left(\int_{0}^{\tau} \alpha(x) d x\right)\right) d \tau}{\int_{t}^{T} \mu^{H}(\tau) \frac{s^{H}(\tau)}{s^{H}(t)} \phi^{\prime}\left(\int_{0}^{\tau} \alpha(x) d x\right) d \tau}$. 
Using assumption A3 and $\phi "<0$, we thus have the following the following inequality:

$\Delta(t) \geq \mu^{L}(t)\left[\frac{\int_{t}^{T} \frac{s^{L}(\tau)}{s^{L}(t)}\left(-\phi^{\prime \prime}\left(\int_{0}^{\tau} \alpha(x) d x\right)\right) d \tau}{\int_{t}^{T} \mu^{L}(\tau) \frac{s^{L}(\tau)}{s^{L}(t)} \phi^{\prime}\left(\int_{0}^{\tau} \alpha(x) d x\right) d \tau}-\frac{\int_{t}^{T} \frac{s^{H}(\tau)}{s^{H}(t)}\left(-\phi^{\prime \prime}\left(\int_{0}^{\tau} \alpha(x) d x\right)\right) d \tau}{\int_{t}^{T} \mu^{L}(\tau) \frac{s^{H}(\tau)}{s^{H}(t)} \phi^{\prime}\left(\int_{0}^{\tau} \alpha(x) d x\right) d \tau}\right]$

Using the following notations:

$$
\begin{aligned}
& g(\tau)=\mu^{L}(\tau) \frac{s^{L}(\tau)}{s^{L}(t)} \phi^{\prime}\left(\int_{0}^{\tau} \alpha(x) d x\right), \\
& k(\tau)=-\frac{\phi^{\prime}\left(\int_{0}^{\tau} \alpha(x) d x\right)}{\phi^{\prime}\left(\int_{0}^{\tau} \alpha(x) d x\right)} \frac{1}{\mu^{L}(\tau)} \\
& h(\tau)=\frac{s^{H}(\tau) / s^{H}(t)}{s^{L}(\tau) / s^{L}(t)}
\end{aligned}
$$

the inequality (17) can be rewritten as:

$$
\Delta(t) \geq \mu^{L}(t)\left[\frac{\int_{t}^{T} g(\tau) k(\tau) d \tau}{\int_{t}^{T} g(\tau) d \tau}-\frac{\int_{t}^{T} g(\tau) k(\tau) h(\tau) d \tau}{\int_{t}^{T} g(\tau) h(\tau) d \tau}\right]
$$

where the functions $g(),. k($.$) and h($.$) are non negative. Rearranging the$ terms in brackets yields:

$\Delta(t) \geq \mu^{L}(t)\left[\frac{\int_{t}^{T} g(\tau) k(\tau) d \tau \int_{t}^{T} g(\tau) h(\tau) d \tau-\int_{t}^{T} g(\tau) k(\tau) h(\tau) d \tau \int_{t}^{T} g(\tau) d \tau}{\int_{t}^{T} g(\tau) d \tau \int_{t}^{T} g(\tau) h(\tau) d \tau}\right]$

where the denominator in brackets is positive. Define the function $f(x)=$ $\int_{t}^{x} g(\tau) k(\tau) d \tau \int_{t}^{x} g(\tau) h(\tau) d \tau-\int_{t}^{x} g(\tau) k(\tau) h(\tau) d \tau \int_{t}^{x} g(\tau) d \tau$. By assumption $\mathrm{A} 2, h$ is non decreasing, $k$ is non increasing since $-\phi^{\prime \prime} / \phi^{\prime}$ is non increasing and $\mu^{L}(\tau)$ is increasing. This implies that $f(x)$ is non decreasing with $x$ and therefore non negative for any $x \geqslant t$. Then $\Delta(t)$ is positive for 
any $t$ which proves the result.

\section{Proof of proposition 1}

The proof is similar to the one provided for Proposition 7 in Bommier (2008).

Differentiating (7) with respect to $t$ yields:

$$
\begin{aligned}
& \dot{\alpha}(t) \beta^{i}(t) u^{\prime}\left(c^{i}(t)\right)+\alpha(t)\left[\dot{c}^{i}(t) u^{\prime \prime}\left(c^{i}(t)\right) \beta^{i}(t)+u^{\prime}\left(c^{i}(t)\right) \dot{\beta}^{i}(t)\right] \\
= & -\lambda \exp \left(-\int_{0}^{t} j(\tau) d \tau\right) j(t)
\end{aligned}
$$

which after some manipulation gives:

$$
\frac{\dot{c}^{i}(t)}{c^{i}(t)}=-\frac{u^{\prime}\left(c^{i}(t)\right)}{u^{\prime \prime}\left(c^{i}(t)\right) c^{i}(t)}\left[j(t)+\frac{\dot{\alpha}(t)}{\alpha(t)}+\frac{\dot{\beta}^{i}(t)}{\beta^{i}(t)}\right]
$$

where $u^{\prime}\left(c^{i}(t)\right) / u^{\prime \prime}\left(c^{i}(t)\right) c^{i}(t)$ is a constant by assumption.

(a) With temporal risk neutrality, $\beta^{i}(t)$ is equal to a constant so that $-\dot{c}^{i}(t) / c^{i}(t)=$ $j(t)+\dot{\alpha}(t) / \alpha(t)$ for $i=H, L$.

(b) With temporal risk aversion, the use of the individual's budget constraint $\int_{0}^{T} c(t) s(t) d t=W_{0}$ and assumption A1 yields $c^{L}(t)>c^{H}(t)$. Finally lemma 1 implies $\dot{c}^{L}(t) / c^{L}(t)<\dot{c}^{H}(t) / c^{H}(t)$. 


\section{Proof of proposition 2}

Differentiation of (9) with respect to $t$ yields:

$\dot{\alpha}^{i}(t)\left[u^{\prime}\left(c^{i}(t)\right) \beta^{i}(t)\right]+\alpha(t)\left[\begin{array}{c}\dot{c}^{i}(t) u^{\prime \prime}\left(c^{i}(t)\right) \beta^{i}(t) \\ +u^{\prime}\left(c^{i}(t)\right) \dot{\beta}^{i}(t)\end{array}\right]=-\lambda \exp \left(-\int_{0}^{t} j(\tau) d \tau\right) j(t)$

which after some manipulation gives:

$$
\frac{\dot{c}^{i}(t)}{c^{i}(t)}=-\frac{u^{\prime}\left(c^{i}(t)\right)}{u^{\prime \prime}\left(c^{i}(t)\right) c^{i}(t)}\left[j(t)+\frac{\dot{\alpha}(t)}{\alpha(t)}+\frac{\dot{\beta}^{i}(t)}{\beta^{i}(t)}\right]
$$

where $u^{\prime}\left(c^{i}(t)\right) / u^{\prime \prime}\left(c^{i}(t)\right) c^{i}(t)$ is a constant by assumption.

(a) With temporal risk neutrality, $\beta^{i}(t)$ is equal to a constant so that (9) implies $c^{i}(t)=c(t)$ for every $t$ and $i=H, L$.

(b) With temporal risk aversion, (9) implies $c^{H}(t)<c^{L}(t)$. Equation (18) and lemma 1 imply $\dot{c}^{L}(t) / c^{L}(t)<\dot{c}^{H}(t) / c^{H}(t)$.

\section{E The pooling optimum}

Differentiating (10) with respect to $t$ yields

$$
\frac{\dot{c}(t)}{c(t)}=-\frac{u^{\prime}(c(t))}{u^{\prime \prime}(c(t)) c(t)}\left[j(t)+\frac{\dot{\bar{\beta}}(t)}{\bar{\beta}(t)}+\frac{\dot{\alpha}(t)}{\alpha(t)}\right]
$$


where

$$
\begin{aligned}
&\left\{\left[n^{H}\left(\dot{s}^{H}(t) \beta^{H}(t)+s^{H}(t) \dot{\beta}^{H}(t)\right)\right.\right. \\
&\left.+n^{L}\left(\dot{s}^{L}(t) \beta^{L}(t)+s^{L}(t) \dot{\beta}^{L}(t)\right)\right]\left[n^{H} s^{H}(t)+n^{L} s^{L}(t)\right] \\
& \frac{\dot{\beta}(t)}{\bar{\beta}(t)}=\frac{\left.-\left[n^{H} s^{H}(t) \beta^{H}(t)+n^{L} s^{L}(t) \beta^{L}(t)\right]\left[n^{H} \dot{s}^{H}(t)+n^{L} \dot{s}^{L}(t)\right]\right\}}{\left[n^{H} s^{H}(t)+n^{L} s^{L}(t)\right]\left[n^{H} s^{H}(t) \beta^{H}(t)+n^{L} s^{L}(t) \beta^{L}(t)\right]}
\end{aligned}
$$

Developing the numerator in the above expression and rearranging terms yields:

$$
\begin{array}{r}
\left\{\left(n^{H} s^{H}(t) \dot{\beta}^{H}(t)+n^{L} s^{L}(t) \dot{\beta}^{L}(t)\right)\left(n^{H} s^{H}(t)+n^{L} s^{L}(t)\right)\right. \\
\frac{\dot{\beta}(t)}{\bar{\beta}(t)}=\frac{\left.+n^{H} n^{L}\left(\beta^{H}(t)-\beta^{L}(t)\right)\left(\dot{s}^{H}(t) s^{L}(t)-\dot{s}^{L}(t) s^{H}(t)\right)\right\}}{\left[n^{H} s^{H}(t)+n^{L} s^{L}(t)\right]\left[n^{H} s^{H}(t) \beta^{H}(t)+n^{L} s^{L}(t) \beta^{L}(t)\right]}
\end{array}
$$

We want to compare $\dot{\bar{\beta}}(t) / \bar{\beta}(t)$ with $\dot{\beta}^{H}(t) / \beta^{H}(t)$ and $\dot{\beta}^{L}(t) / \beta^{L}(t)$.

Let first denote $\Lambda$, the difference between $\dot{\bar{\beta}}(t) / \bar{\beta}(t)$ and $\dot{\beta}^{H}(t) / \beta^{H}(t)$. It has the following expression

$$
\begin{array}{r}
\left\{n^{L} s^{L}(t)\left(n^{H} s^{H}(t)+n^{L} s^{L}(t)\right)\left(\dot{\beta}^{L}(t)-\beta^{L}(t) \frac{\dot{\beta}^{H}(t)}{\beta^{H}(t)}\right)\right. \\
\Lambda=\frac{\left.+n^{H} n^{L}\left(\beta^{H}(t)-\beta^{L}(t)\right)\left(\dot{s}^{H}(t) s^{L}(t)-\dot{s}^{L}(t) s^{H}(t)\right)\right\}}{\left[n^{H} s^{H}(t)+n^{L} s^{L}(t)\right]\left[n^{H} s^{H}(t) \beta^{H}(t)+n^{L} s^{L}(t) \beta^{L}(t)\right]}
\end{array}
$$

which is always negative by Assumption A1 and Lemma 1. This implies $\stackrel{\bar{\beta}}{\beta}(t) / \bar{\beta}(t)<\dot{\beta}^{H}(t) / \beta^{H}(t)$.

Let us now denote $\Upsilon$ the difference between $\dot{\bar{\beta}}(t) / \bar{\beta}(t)$ and $\dot{\beta}^{L}(t) / \beta^{L}(t)$. 
It yields:

$$
\Upsilon=\frac{n^{H} s^{H}(t)\left\{\left(n^{H} s^{H}(t)+n^{L} s^{L}(t)\right)\left(\dot{\beta}^{H}(t)-\beta^{H}(t) \frac{\dot{\beta}^{L}(t)}{\beta^{L}(t)}\right)\right.}{\left[n^{H} s^{H}(t)+n^{L} s^{L}(t)\right]\left[n^{H} s^{H}(t) \beta^{H}(t)+n^{L} s^{L}(t) \beta^{L}(t)\right]}
$$

where the first part in the numerator is positive while the second one is negative. Equivalently,

$$
\begin{aligned}
& \Upsilon=\chi\left\{\mu^{H}(t)\left(\frac{\dot{\beta}^{H}(t)}{\mu^{H}(t)}\left(n^{H} s^{H}(t)+n^{L} s^{L}(t)\right)+n^{L} s^{L}(t)\left[\beta^{L}(t)-\beta^{H}(t)\right]\right)\right. \\
& \left.-\mu^{L}(t)\left(\frac{\beta^{H}(t)}{\beta^{L}(t)} \frac{\dot{\beta}^{L}(t)}{\mu^{L}(t)}\left(n^{H} s^{H}(t)+n^{L} s^{L}(t)\right)+n^{L} s^{L}(t)\left[\beta^{L}(t)-\beta^{H}(t)\right]\right)\right\}
\end{aligned}
$$

with $\chi=n^{H} s^{H}(t) /\left[\left(n^{H} s^{H}(t)+n^{L} s^{L}(t)\right)\left(n^{H} s^{H}(t) \beta^{H}(t)+n^{L} s^{L}(t) \beta^{L}(t)\right)\right]>$ 0 . First note that, using equation (16), $\dot{\beta}^{i}(t)$ can be rewritten as

$$
\dot{\beta}^{i}(t)=\mu^{i}(t)\left(\beta^{i}(t)-\phi^{\prime}\left(\int_{0}^{t} \alpha(x) d x\right)\right)
$$

where $\beta^{i}(t)-\phi^{\prime}\left(\int_{0}^{t} \alpha(x) d x\right)<0$. Using this expression and rearranging terms, this yields:

$$
\begin{aligned}
& \frac{\dot{\beta}^{H}(t)}{\mu^{H}(t)}\left(n^{H} s^{H}(t)+n^{L} s^{L}(t)\right)+n^{L} s^{L}(t)\left[\beta^{L}(t)-\beta^{H}(t)\right]= \\
& n^{H} s^{H}(t)\left(\beta^{H}(t)-\phi^{\prime}\left(\int_{0}^{t} \alpha(x) d x\right)\right)+n^{L} s^{L}(t)\left(\beta^{L}(t)-\phi^{\prime}\left(\int_{0}^{t} \alpha(x) d x\right)\right)
\end{aligned}
$$


which is always negative. Using A1 and Lemma 1, one also has

$$
\begin{aligned}
& \frac{\beta^{H}(t)}{\beta^{L}(t)} \frac{\dot{\beta}^{L}(t)}{\mu^{L}(t)}\left(n^{H} s^{H}(t)+n^{L} s^{L}(t)\right)+n^{L} s^{L}(t)\left[\beta^{L}(t)-\beta^{H}(t)\right] \\
< & \frac{\dot{\beta}^{H}(t)}{\mu^{H}(t)}\left(n^{H} s^{H}(t)+n^{L} s^{L}(t)\right)+n^{L} s^{L}(t)\left[\beta^{L}(t)-\beta^{H}(t)\right]<0
\end{aligned}
$$

so that $\Upsilon>0$ and $\dot{\beta}^{L}(t) / \beta^{L}(t)<\dot{\bar{\beta}}(t) / \bar{\beta}(t)$.

Using expressions $(18)$ and $\dot{\beta}^{L}(t) / \beta^{L}(t)<\overline{\bar{\beta}} / \bar{\beta}(t)<\dot{\beta}^{H}(t) / \beta^{H}(t)$ yields:

$$
{\frac{\dot{c}^{L}(t)}{c^{L}(t)}}^{F B}<\frac{\dot{c}(t)}{c(t)}<{\frac{\dot{c}^{H}(t)}{c^{H}(t)}}^{F B}
$$

which proves the result.

\section{F Second best optimum}

\section{F.1 Proof of point (ii) of proposition 3}

First order conditions of the second best problem are:

$$
\begin{aligned}
& \frac{\partial E U^{H}}{\partial c^{H}(t)}\left(1+\frac{\gamma}{n^{H}}\right)-\lambda s^{H}(t) \exp \left(-\int_{0}^{t} j(\tau) d \tau\right)=0 \\
& \frac{\partial E U^{L}}{\partial c^{L}(t)}-\lambda s^{L}(t) \exp \left(-\int_{0}^{t} j(\tau) d \tau\right)-\frac{\gamma}{n^{L}} \frac{\partial E U^{H}}{\partial c^{L}(t)}=0 .
\end{aligned}
$$

(a) Straightforward rearrangement of (20) taken at time $t$ and $t+\epsilon$ gives the marginal rate of substitution between two-period consumptions (11) for the high type individual which proves point (a). 
(b) Denoting $E U_{c(t)}^{j}$ the expected marginal utility of consumption at date $t$ and evaluating (21) at time $t$ and $t+\epsilon$, we get:

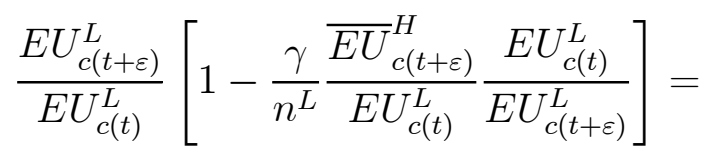

$$
\begin{aligned}
& \frac{s^{L}(t+\varepsilon)}{s^{L}(t)} \exp \left(-\int_{0}^{t} j(\tau) d \tau\right) \times\left[1-\frac{\gamma}{n^{L}} \frac{\overline{E U_{c(t)}^{H}}}{E U_{c(t)}^{L}}\right]
\end{aligned}
$$

where $\overline{E U}_{c(t)}^{H}$ is the expected marginal utility of a type $H$ individual mimicking a type $L$ individual. Multiplying the second term in brackets of the LHS by $\overline{E U}_{c(t)}^{H} / \overline{E U}_{c(t)}^{H}$ yields:

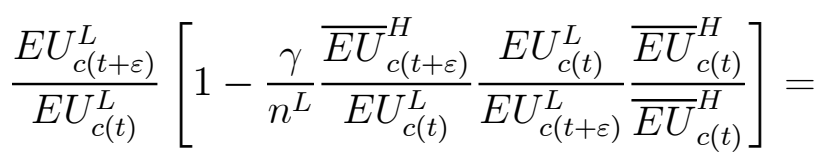

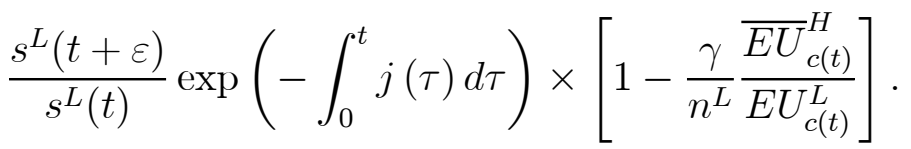

This can be rewritten as:

$$
\begin{aligned}
& M R S_{c(t), c(t+\varepsilon)}^{L}\left[1-\frac{\gamma}{n^{L}} \frac{\overline{M R S_{c(t), c(t+\varepsilon)}^{H}}}{M R S_{c(t), c(t+\varepsilon)}^{L}} \frac{s^{H}(t) \beta^{H}(t)}{s^{L}(t) \beta^{L}(t)}\right]= \\
& \frac{s^{L}(t+\varepsilon)}{s^{L}(t)} \exp \left(-\int_{0}^{t} j(\tau) d \tau\right) \times\left[1-\frac{\gamma}{n^{L}} \frac{s^{H}(t) \beta^{H}(t)}{s^{L}(t) \beta^{L}(t)}\right]
\end{aligned}
$$

which yields (12). 
(c) The first order conditions (20) and (21) can be rewritten as:

$$
\begin{gathered}
\alpha(t) u^{\prime}\left(c^{H}(t)\right) \beta^{H}(t)\left(1+\frac{\gamma}{n^{H}}\right)=\lambda \exp \left(-\int_{0}^{t} j(\tau) d \tau\right), \\
\alpha(t) u^{\prime}\left(c^{L}(t)\right)\left[\beta^{L}(t)-\frac{\gamma}{n^{L}} \frac{s^{H}(t)}{s^{L}(t)} \beta^{H}(t)\right]=\lambda \exp \left(-\int_{0}^{t} j(\tau) d \tau\right) .
\end{gathered}
$$

Differentiating (22) with respect to time yields $\dot{c}^{H}(t) / c^{H}(t)$ to be the same as in (18) whereas differentiation of (23) yields:

$$
\begin{aligned}
& \frac{\dot{c}^{L}(t)}{c^{L}(t)}=-\frac{u^{\prime}\left(c^{L}(t)\right)}{c^{L}(t) u^{\prime \prime}\left(c^{L}(t)\right)} \times \\
& \left(j(t)+\frac{\dot{\alpha}(t)}{\alpha(t)}+\frac{\dot{\beta}^{L}(t)-\gamma\left(\frac{s^{H}(t)}{s^{L}(t)} \dot{\beta}^{H}(t)+\left(\frac{\dot{s}^{H}(t)}{s^{L}(t)}-\frac{\dot{s}^{L}(t)}{s^{L}(t)} \frac{s^{H}(t)}{s^{L}(t)}\right) \beta^{H}(t)\right)}{\beta^{L}(t)-\gamma \frac{s^{H}(t)}{s^{L}(t)} \beta^{H}(t)}\right) \\
= & -\frac{u^{\prime}\left(c^{L}(t)\right)}{c^{L}(t) u^{\prime \prime}\left(c^{L}(t)\right)} \times \\
& \left.j(t)+\frac{\dot{\alpha}(t)}{\alpha(t)} \cdot+\frac{\dot{\beta}^{L}(t)}{\beta^{L}(t)}\left[\frac{1-\gamma\left(\frac{s^{H}(t)}{s^{L}(t)} \frac{\dot{\beta}^{H}(t)}{\dot{\beta}^{L}(t)}+\left(\frac{\dot{s}^{H}(t)}{s^{L}(t)}-\frac{\dot{s}^{L}(t)}{s^{L}(t)} \frac{s^{H}(t)}{s^{L}(t)}\right) \frac{\beta^{H}(t)}{\dot{\beta}^{L}(t)}\right)}{1-\gamma \frac{s^{H}(t)}{s^{L}(t)} \frac{\beta^{H}(t)}{\beta^{L}(t)}}\right] 4 .\right)
\end{aligned}
$$

Note that by assumption A2 and lemma 1, one has:

$$
\frac{\dot{s}^{H}(t)}{s^{H}(t)}+\frac{\dot{\beta}^{H}(t)}{\beta^{H}(t)}>\frac{\dot{\beta}^{L}(t)}{\beta^{L}(t)}+\frac{\dot{s}^{L}(t)}{s^{L}(t)}
$$

so that

$$
\left(\frac{s^{H}(t)}{s^{L}(t)} \frac{\dot{\beta}^{H}(t)}{\dot{\beta}^{L}(t)}+\left(\frac{\dot{s}^{H}(t)}{s^{L}(t)}-\frac{\dot{s}^{L}(t)}{s^{L}(t)} \frac{s^{H}(t)}{s^{L}(t)}\right) \frac{\beta^{H}(t)}{\dot{\beta}^{L}(t)}\right)>\frac{s^{H}(t)}{s^{L}(t)} \frac{\beta^{H}(t)}{\beta^{L}(t)}
$$


which implies

$$
\frac{1-\gamma\left(\frac{s^{H}(t)}{s^{L}(t)} \frac{\dot{\beta}^{H}(t)}{\dot{\beta}^{L}(t)}+\left(\frac{\dot{s}^{H}(t)}{s^{L}(t)}-\frac{\dot{s}^{L}(t)}{s^{L}(t)} \frac{s^{H}(t)}{s^{L}(t)}\right) \frac{\beta^{H}(t)}{\dot{\beta}^{L}(t)}\right)}{1-\gamma \frac{s^{H}(t)}{s^{L}(t)} \frac{\beta^{H}(t)}{\beta^{L}(t)}}>1 .
$$

Thus, comparing (24) with (18) yields $\left(\dot{c}^{L}(t) / c^{L}(t)\right)^{S B}<\left(\dot{c}^{L}(t) / c^{L}(t)\right)^{F B}$.

\section{F.2 Implementation}

For $\epsilon$ small enough, (13) can be rewritten as:

$$
\left(1-\epsilon r^{L}(t)\right)\left(1-\pi(t) \frac{\overline{M R S}_{c(t), c(t+\epsilon)}^{H}}{M R S_{c(t), c(t+\epsilon)}^{L}}\right)=\left(1-\epsilon\left(j(t)+\mu^{L}(t)\right)\right)(1-\pi(t))
$$

Rearranging the terms yields:

$$
\begin{aligned}
& \left(1-\epsilon r^{L}(t)\right)\left[M R S_{c(t), c(t+\varepsilon)}^{L}-\pi(t) \overline{M R S}_{c(t), c(t+\epsilon)}^{H}\right] \\
= & \left(1-\epsilon\left(j(t)+\mu^{L}(t)\right)\right)(1-\pi(t)) M R S_{c(t), c(t+\varepsilon)}^{L} .
\end{aligned}
$$

Using (14), one has:

$$
M R S_{c(t), c(t+\epsilon)}^{i}=1+\epsilon \Omega^{i}
$$

where $\Omega^{i}=\left[\frac{\dot{s}^{i}(t)}{s^{i}(t)}+\frac{\dot{\alpha}(t)}{\alpha(t)}+\frac{\dot{\beta}^{i}(t)}{\beta^{i}(t)}+\frac{u^{\prime \prime}(c(t))}{u^{\prime}(c(t))} \dot{c}(t)\right]$ so that (25) can be rewritten as:

$$
\begin{aligned}
& \left(1-\epsilon r^{L}(t)\right)\left[(1-\pi(t))+\epsilon\left(\Omega^{L}-\pi(t) \Omega^{H}\right)\right] \\
= & \left(1-\epsilon\left(j(t)+\mu^{L}(t)\right)\right)(1-\pi(t))\left(1+\epsilon \Omega^{L}\right) .
\end{aligned}
$$


After some simplification, this yields for $\epsilon$ small enough:

$$
\left[r^{L}(t)-\left(j(t)+\mu^{L}(t)\right)\right]=\frac{\pi(t)}{(1-\pi(t))}\left(\Omega^{L}-\Omega^{H}\right)
$$

which gives (15). 